\title{
Design Rules of Hybrid Stepping Machine for Free Piston Engine
}

\author{
Sung-In Jeong ${ }^{\dagger}$
}

\begin{abstract}
This paper presents the hybrid stepping machine for linear oscillating generators. The focus of the work is the suggestion of the improved model through the comparison of proposed models ; new flux concentrating PMs mover of the hybrid stepping generator is proposed based on the symmetrical and non-symmetrical stator cores of the surface mounted PMs mover, and non-slanted PMs and slanted PMs of the flux concentrating PMs mover. It is achieved using equivalent magnetic circuit considering leakage elements. Finally, this study suggests new hybrid stepping structure of linear oscillating generator.
\end{abstract}

Keywords: HSPM, IC (Internal Combustion), Linear oscillating generator, Free piston engine, Leakage reluctances, Equivalent magnetic circuit, Flux concentrating, FEA

\section{Introduction}

The linear PM brushless oscillatory generators have gained momentum recently, HEVs [1]. Most of the research on direct drive application such as HEVs has focused on variable reluctance PM machines, transverse flux PM machines or hybrid stepping machines. The leading feature of these machines is the very high force-to-mass ratio compared with classical machines, which makes them attractive in low speed applications by reason of high pole number. The moving piston of this generator will be directly coupled to rod. Similarly, the flux path of a hybrid stepping machine is longitudinal, or parallel to the direction of movement; it is distinguished from transverse flux that flux lines go perpendicular to the moving of translator.

The generator will be used for direct-drive power takeoff in an IC engine. This develops an analytical model for a new double-sided hybrid stepping linear machine for use in IC engine applications. So, the intent of this study is to introduce a new type of Hybrid Stepping Permanent Magnet (HSPM) linear machine of single-phase for possible use as a direct-drive in future IC engine.

Hybrid stepping machine is a doubly-salient machine with magnets on the mover. The flux linkage by phase concentrated coils in the stator reverses polarity with the traveling of the mover. It has numerous advantages such as simple construction, low inertia and high force density application due to PMs on the mover pole. The stator also offers a large freedom in winding configurations, which is an important tool to reduce cogging forces. The stator winding is made of standard wires with circular crosssection. Furthermore, conductors with circular crosssection made of several strands are very flexible and simplify the winding process of the stator considerably.

This study investigates how different design choices

$\dagger$ Corresponding Author: R\&D Center, Daelim Motor, Korea.

(in8130@hanyang.ac.kr)

Received: November 29, 2016; Accepted: March 6, 2017

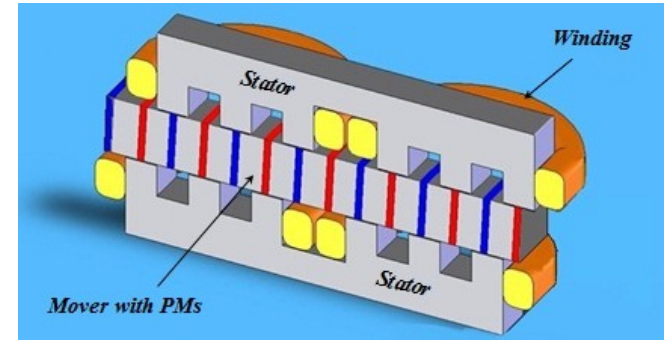

Fig. 1. Hybrid stepping permanent magnet machine

affect the performance of the HSPM generator. As the factors affecting, it is considered by types of magnets. This is an important design variable which determines force performance in given stator tooth. Henceforth, it will be performed in analytical calculation by equivalent magnetic circuit method considering leakage and also characteristics simulated based on finite element formulation.

The schematic diagram of the hybrid stepping permanent magnet generator is shown Fig. 1. It consists of the stator with single-phase winding iron core of upper and lower part with single-phase winding, and the mover with magnets between iron cores of middle part.

\section{Proposed Topologies}

In this present work two concepts of magnet fixation; surface mounted magnets and flux concentrating magnets between translator pole shoes have been studied with $\mathrm{NdFeB}$. The topologies are composed of a primary (stator) and a secondary (translator) with surface mounted magnets and flux concentrating magnets. The translator is sandwiched between two stators that carry flux in the longitudinal direction, while the translator carries flux in the transverse direction. Generally, linear oscillating generator refers to that performs a linear motion with a certain stroke at a specific. 
This proposes a suitable generator for IC engine; it is hybrid stepping machine which has the advantages of high specific force capability. Therefore, the different configurations are compared with respect to shape of magnets as follows.

- Surface Mounted PMs Mover

- Symmetrical Stator Cores

- Non Symmetrical Stator Cores

- Flux Concentrating PMs Mover

- Non Slanted PMs

- Slanted PM

- New Flux Concentrating PM Mover

The surface mounted PMs mover model facilitates more mechanically stable drive because iron-core absorbs the shock from the piston, consequently the impulse of magnets shocked directly is small. However, there is a disadvantage in high-speed operation characteristics by increasing of iron-core amount as compared with the flux concentrating PMs mover model. On the other hand, the flux concentrating PMs mover model is suitable for highspeed operation by light weight of iron-core amount, but even it can happen to risk of damage to magnets owing to the shock of piston.

\subsection{Surface mounted PMs mover}

This topology has two armatures of upper and lower part with surface mounted magnets mover. The mover consists of 14 magnets which magnetized vertical direction in top and bottom of mover iron-core, respectively. However, it has a disadvantage structurally because the mover suffers from a high-speed oscillating motion; it can be easily separated from the mover iron during the motion or an

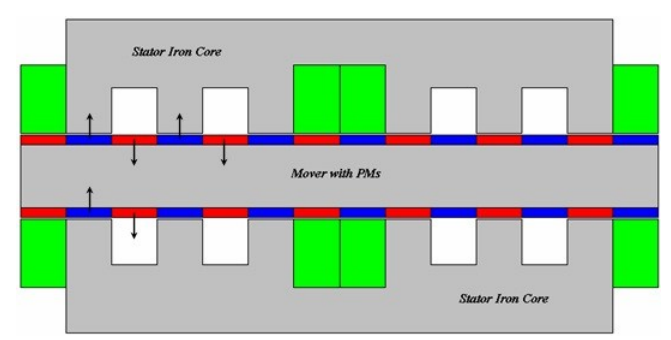

(a) Symmetry stator-cores

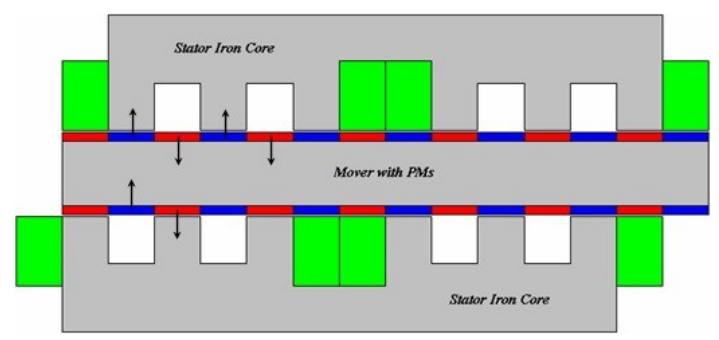

(b) Asymmetry stator-cores

Fig. 2. HSPM machine with surface mounted PMs mover adhesive part to fix the magnet to mover can be damaged [2]. The stator is made of non-oriented laminated electrical steel with thin insulating coating.

Fig. 2 shows HSPM machine with surface mounted magnets mover which magnetized $y$-axis; one is the stator cores consisted of symmetrical structure each other, and the other are misaligned by one pole-pitch of the stator. The mover is made of an iron core with attached $\mathrm{NdFeB}$ which is fixed at $10[\mathrm{~mm}]$ of width per one-pole in cross section area. In the Fig. 2, the flux concentration in the symmetry stator-cores model does not happen in the mover because two armatures of upper and lower part are symmetrical. Meanwhile, the flux in case of the asymmetry stator-cores model is concentrated in the mover because the flux exists in both transverse and longitudinal form simultaneously due to asymmetry of upper and lower stator core.

This will be discussed in further in paragraph 4 Comparison Results.

\subsection{Flux concentrating PMs mover}

A different longitudinal rectangular shape of the magnets is investigated with respect to the magnetic flux. As seen from the Fig. 3, the magnets should be as long as possible for the chosen volume to maximize the magnetic flux in the circuit. The NdFeB has a relatively high coercivity and the height of magnet can thus be kept small without reducing the magnetic field intensity due to a high demagnetizing field inside the magnets. In this way, increased magnetic flux implies smaller magnet size. The flux concentrating PMs mover model has some good points, such as it can prevent an adhesive part from being removed and damaged. It also indicates the conceptual design of two

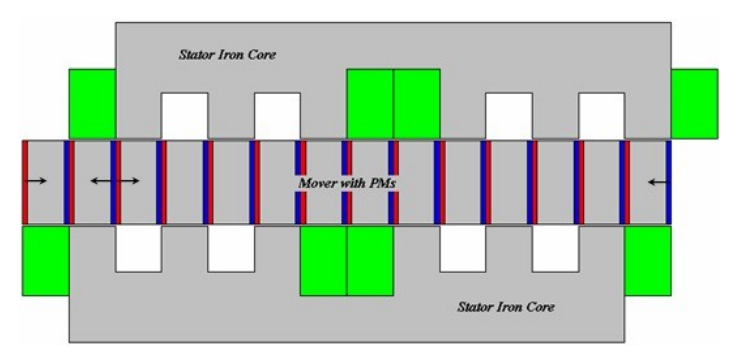

(a) Non slanted permanent magnets

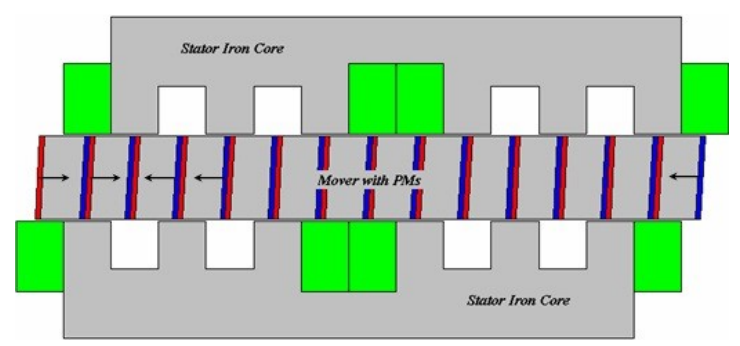

(b) Slanted permanent magnets

Fig. 3. HSPM machine with flux concentrating PMs mover 
models; non-slanted magnets and slanted magnets in flux concentrating PMs mover model as shown in Fig. 3. The cross-section area of overall mover including magnets in the flux concentrating PMs mover model is equal to that in the surface mounted PMs mover model. It is only different in magnetization direction of the magnets compared to the surface mounted PMs mover model. In addition, the crosssection area of magnet per pole in the flux concentrating PMs mover model is $18.9\left[\mathrm{~mm}^{2}\right]$; this is smaller than that of the surface mounted PMs mover model of $20\left[\mathrm{~mm}^{2}\right]$.

Consequently, the flux concentrating PMs mover model has the grab on the weight of mover and material cost aspects. In order to improve flux linkage, the evaluation in all flux concentrating PMs mover models is considered using the same amount of material such as iron steel, conductor and magnets as well as same input current and remanence. This makes it possible to concentrate a flux; as a result we can expect the performances that the flux concentrating PMs mover model leads to more improved results than the surface mounted PMs mover model. This scientific background will be presented in next section.

\section{Analytical Calculation}

This HSPM topology mentioned at the beginning is inevitable to reduce the force ripple in the electromagnetic force due to the frequent variation of reluctance between stator teeth and translator with magnets. It needs in-depth investigation to optimize the use of expensive magnets and other materials, minimizing force ripple through analyses of the magnetic circuit. Either, HSPM generator is the existence of end of winding, resulting in low power factor. End winding are undesired as they increase the cost of the device and the stator resistance of each phase without proving a useful advantages. By modeling this generator, the effect of longitudinal leakage flux at the stator ends is neglected. Since there are so many poles, this effect is minimal [3]. The following numerical process is used in the design of the HSPM generator.

- Use the leakage reluctance in air region and the flux linkage per pole by electromagnetic force

- Now that we have all the machine dimensions, we can find the average force and force density using the stored magnetic energy

FEA is used to evaluate and compare to the analytical results of proposed types as well as calculate leakage reluctance and flux linkage.

\section{Comparison Results}

\subsection{Surface mounted PMs mover}

A flux characteristic of a surface mounted PMs mover

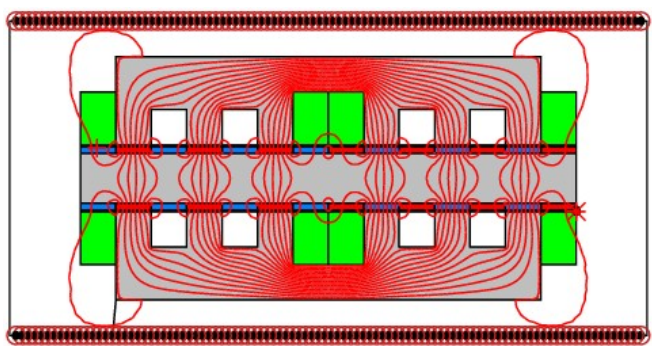

(a) Symmetry stator-cores

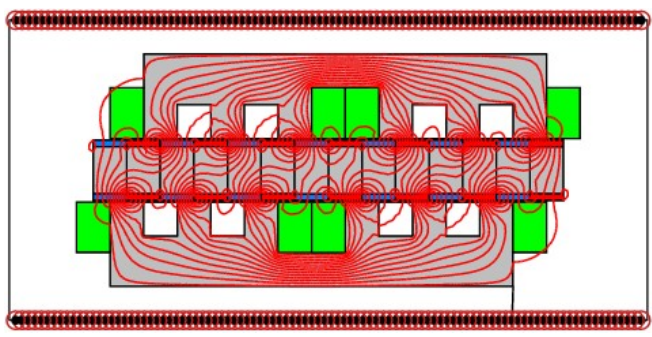

(b) Asymmetry stator-cores

Fig. 4. Flux characteristic of surface mounted PMs mover machine

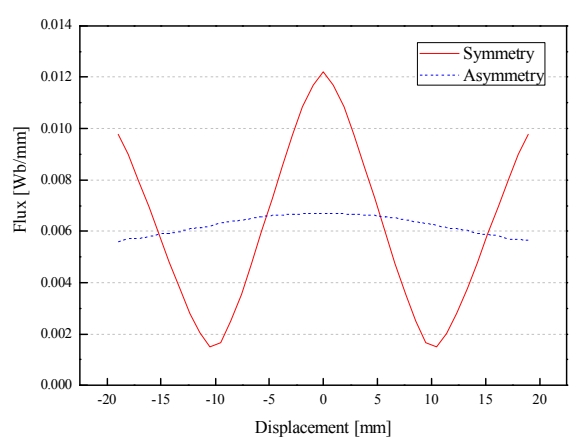

(a) Flux profile

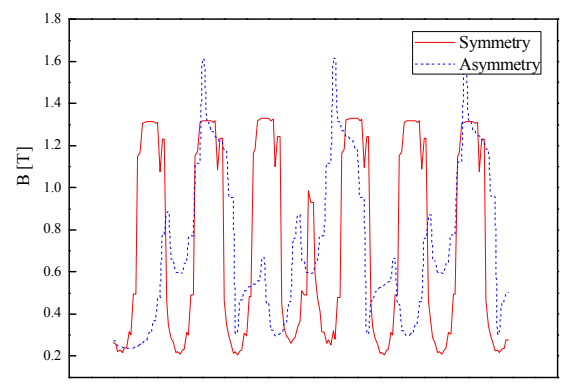

(b) Flux density in air-gap

Fig. 5. Surface mounted PMs mover machine

generator is shown in Fig. 4. Aligned stator core type each other lead to increase leakage flux in the end part as called end effect, while the flux feature of misaligned stator core type shows larger leakage flux in air regions of stator relatively. It is an inevitable phenomenon in such a structure since the magnets are magnetized by vertical direction with rectangular shape. 


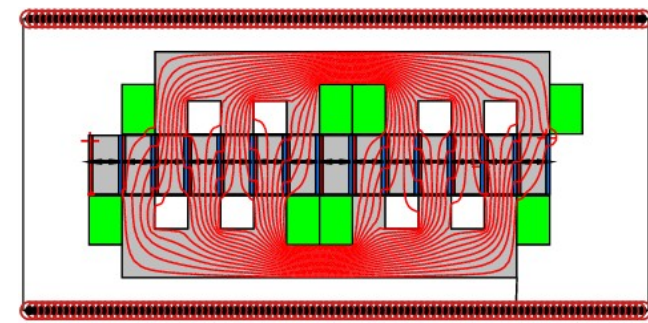

(a) Non slanted PMs

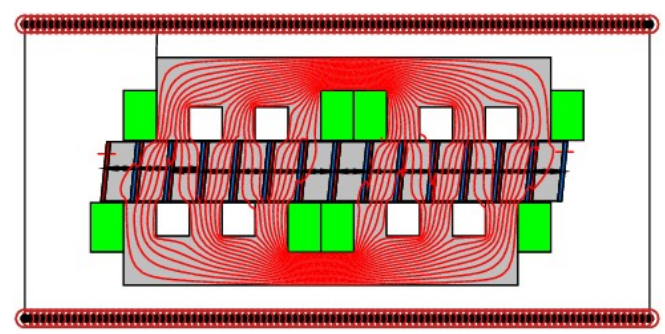

(b) Slanted PMs

Fig. 6. Flux characteristic of flux concentrating PMs mover machine

By FE analysis of two different types, the flux characteristics are shown in Fig. 5; the flux features various from symmetric form of upper and lower stator cores. The solid line represents the flux profile of the symmetrical stator cores model, whereas the dotted line relates to the flux curve of the asymmetrical stator cores model. The flux linkage of the symmetrical stator cores model shows the better result than that of the asymmetrical stator cores model because the symmetrical stator cores model has a steady flux density in the air-gap.

\subsection{Flux concentrating PMs mover}

The flux performances of the flux concentrating PMs mover generator with the non-slanted and slanted magnets are represented in Fig. 6.

The slanting angle versus longitude direction of the shaft has a very large effect on the cogging force. By slanting the magnets, it is effective in reducing the cogging force as well as expected to improve force [4]. All of HSPM machines with the flux concentrating PMs mover are shifted as one pole-pitch of the stator.

Cogging is not unique to linear generators; rotary machines have the same problem and there are several methods traditionally used to minimize the effect that can also be employed in linear generators [5].

These include skewing the magnets, shaping of the magnets, and / or skewing the slots in the laminations. All of these reduce cogging by intentionally misaligned the laminations and magnets. This softens the transitions of the laminations teeth from one magnet to the next magnet and reduces cogging. Therefore, the design study will be considered about relationship between force characteristic

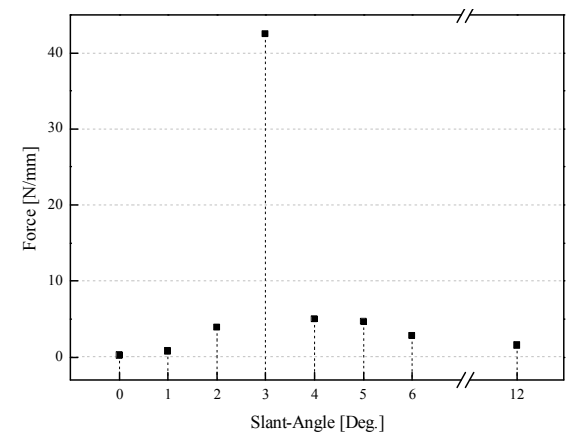

(a) Force by slant-angle

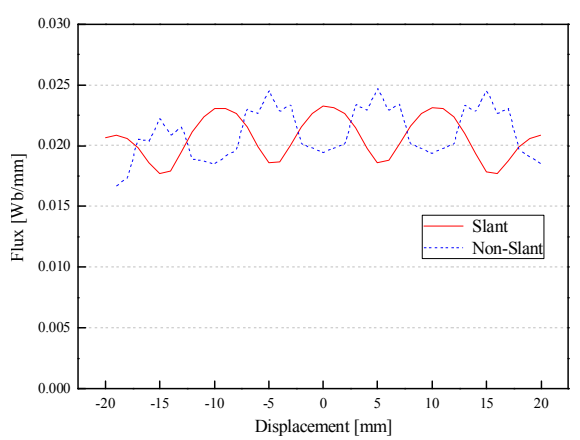

(b) Flux profile of slant and non-slant

Fig. 7. Flux concentrating PMs mover machine

and slant-angle of magnet to minimize cogging.

Generally, slanting the mover with magnets will also adversely affect the flux linkage. However, there exists an optimum slant-angle that greatly reduces the cogging force but only slightly reduces the flux linkage variation. The force variation by slant-angle of magnets is investigated varying from $1[\mathrm{Deg}$.$] to 6[\mathrm{Deg}$.$] at interval 1$ [Deg.] and 12 [Deg.] of mechanical degree. This result of the study brings to the important solution to find the optimal slantangle to minimize cogging force, thereby improving feature of the force. In analysis of slant-angle change from non-slant to $12[\mathrm{Deg}$.$] , the force value is the largest at$ 3 [Deg.] of slant-angle; non-slanted magnet means zero [Deg.] in slant-angle.

The simulated results show the significant difference in force characteristic for different slant-angle of magnets as shown Fig. 7. The flux performance in slanted magnets model is smoother than non-slanted magnets model. Here, it is clearly illustrated how the shape of the magnets affects the flux path in the magnetic circuit significantly.

\section{Selection of Surface Mounted and Flux Concentrating PMs Mover Types}

As mentioned earlier, the sectional area of magnet in the flux concentrating PMs mover type is smaller than that of the surface mounted PMs mover type. All of two types, non-slanted and slanted magnets of flux concentrating PMs 
mover, the cross-sectional area of magnet per pole is $10.5\left[\mathrm{~mm}^{2}\right]$. Decreasing the width of magnets causes the reduction of the equivalent current density of the magnets. It has more effects; one of the most important effects is enhancing the generator output voltage.

However, this is not always good, especially when harmonics and peaky components appear in the output voltage. Generally, the flux concentrating PMs mover type shows better characteristics in terms of weight of mover and electrical performances such as the flux linkage and force. The proposed flux concentrating PMs mover type for flux concentration has some good points, such as the flux linkage is large considerably because the magnetic flux can be concentrated effectively by structure of buried magnet inside mover.

Fig. 8 indicates the comparison of measured flux performances for the symmetry, asymmetry structures in the surface mounted PMs mover type and non-slanted, slanted in the flux concentrating PMs mover type. It can be observed that the characteristics of the flux concentrating PMs mover types are in good agreement with that of the surface mounted PMs mover types in view of the flux linkage and cogging force. Compared to the symmetry stator cores structure of the surface mounted PMs mover type, magnetic flux value of the slanted magnets type in case of the aligned position is almost twice bigger. Also, the cogging force in the flux concentrating PMs mover types shows positive results than that of the surface mounted PMs mover type. Particularly, it can be seen that

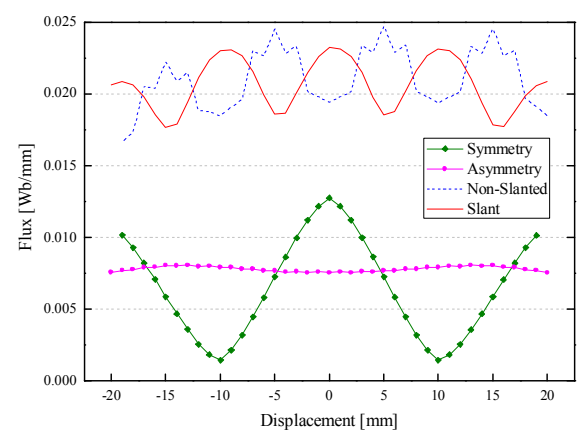

(a) Flux profiles

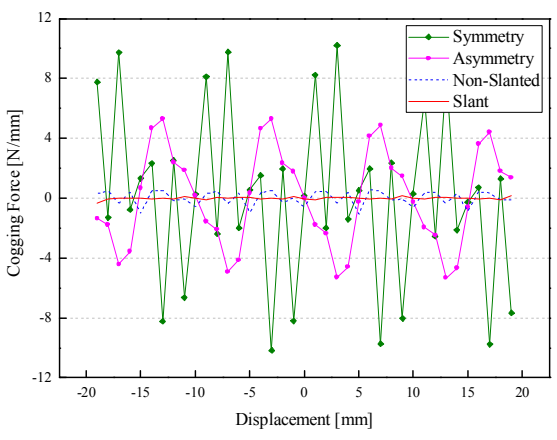

(b) Cogging force

Fig. 8. Comparison of categories the cogging force is reduced considerably in the slanted PMs mover of flux concentrating PMs mover type.

In conclusion, the flux concentrating PMs mover type can not only concentrate magnetic flux, but also can obtain the soft flux performance and lower cogging force by slanting of magnets. As a result, it gives information that the slanted magnets of $3[\mathrm{Deg}$.] in the flux concentrating PMs mover type have the best force performance among different four models.

\section{Proposal Model}

As stated above, the flux concentrating PMs mover type is more effective than the surface mounted PMs mover type because it obtains bigger magnetic flux and larger force relatively. Furthermore, the change by slant-angle of magnets seriously affects force performance; as a result, it could achieve the best result at 3[Deg.] of slant-angle in force aspects.

Ultimately, this chapter suggests new hybrid stepping structure of linear oscillating generator as shown Fig. 9 [6]; the cross-sectional area of magnet per pole is identical to the flux concentrating PMs mover type, accordingly the whole size of magnets is half of the flux concentrating PMs mover type.

Therefore, it can be expected to bring about better results in respect of force performance by high-speed operation one of the advantages of linear machines. In addition, this topology will be analyzed through comparing the flux concentrating slanted PMs mover type by analytical and numerical calculation.

\subsection{Analytical calculation of proposed model}

The newly proposed hybrid stepping generator is calculated by dividing maximum and minimum magnetic energy.
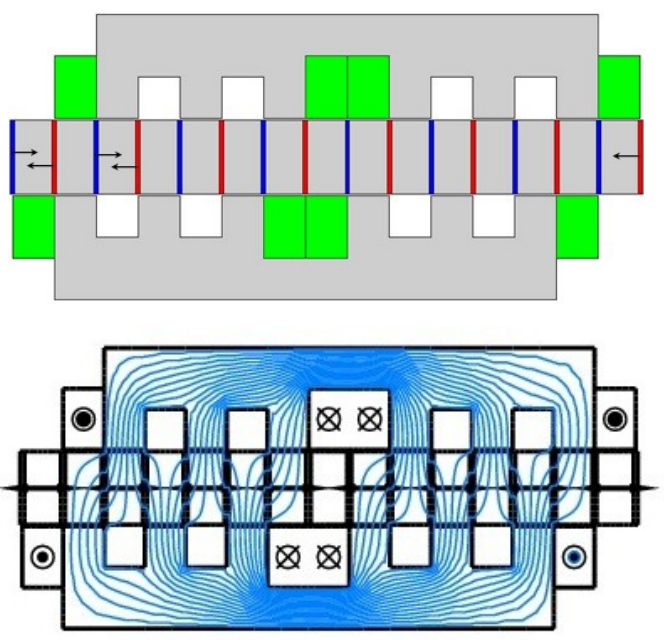

Fig. 9. Proposed model 
Fig. 10 shows the structure and equivalent magnetic circuit of hybrid stepping generator for magnetic energy calculation.

Analytical expression for the force and magnetic energy calculation are as follows.

$\theta_{a}=N_{c} \cdot I$

$\theta_{m}=B_{r e m} \cdot h_{m} / \mu_{0} \cdot \mu_{r}$

The reluctances for magnetic energy calculation are expressed as Eqs. (3), (4) and (5). This indicates the reluctance in air-gap, magnet and leakage, respectively. The $B_{\text {rem }}$ represents residual magnetic flux density (remanence) of magnet.

$R_{\delta}=1 / \mu_{0} \cdot 2 \cdot \delta / l_{z} \cdot w_{t}$

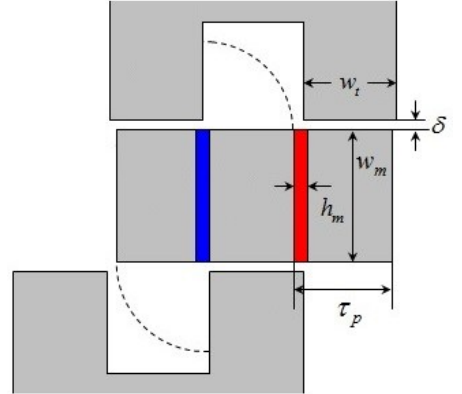

(a) Arrangement where, $l_{z}$ indicates the active length of $z$-axis direction.

$$
\begin{aligned}
& R_{m}=1 / \mu_{0} \cdot \mu_{r} \cdot h_{m} / l_{z} \cdot w_{m} \\
& R_{\sigma}=1 / \mu_{0} \cdot h_{m} / l_{z} \cdot\left(\tau_{p}-h_{m}\right)+1 / \mu_{0} \cdot \pi / 2 \cdot 1 / \\
& \ln \left(\left(\tau_{p}-h_{m}\right) / \delta\right) \\
& \emptyset_{a \delta}=\theta_{a} \cdot\left(2 \cdot R_{\sigma}+R_{m}\right) / 2 \cdot R_{\delta} \cdot R_{\sigma}+\left(R_{\sigma}+R_{\delta}\right) \cdot R_{m} \\
& \emptyset_{a \sigma}=\theta_{a} \cdot(2) \\
& \left.\emptyset_{m \delta}=2 \cdot \theta_{\delta}+R_{m}\right) / 2 \cdot R_{\delta} \cdot R_{\sigma}+\left(R_{\sigma}+R_{\delta}\right) \cdot R_{m} \\
& \emptyset_{m \sigma}=2 \cdot R_{\delta} \cdot R_{\sigma}+\left(R_{\sigma}+R_{\delta}\right) \cdot R_{m}
\end{aligned}
$$

The total magnetic energy can be obtained using the difference between the minimum and maximum magnetic energy as follows.

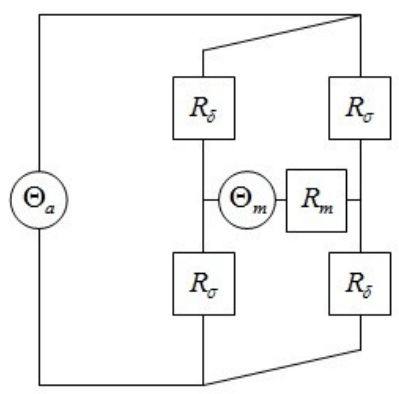

(b) Equivalent magnetic circuit

Fig. 10. Configuration of new hybrid stepping generator

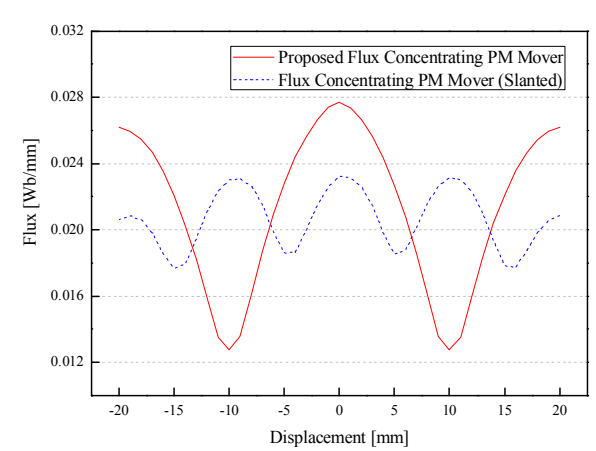

(a) Flux profile

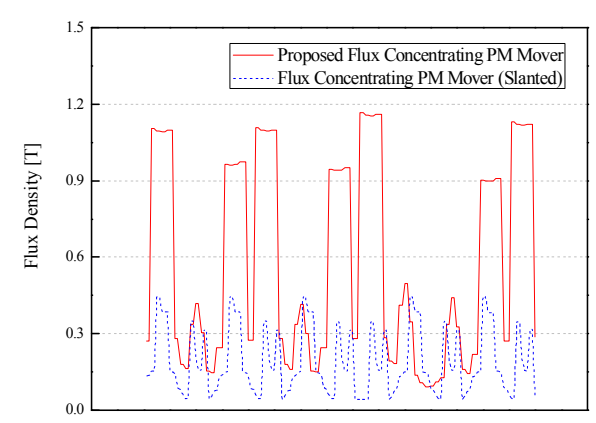

(c) Flux density in air-gap (no-load)

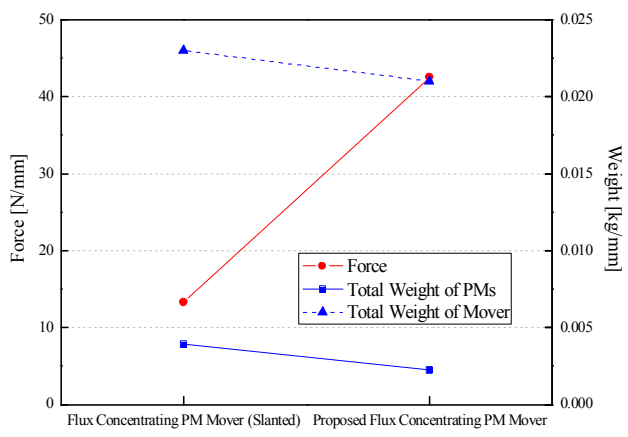

(b) Force and weight

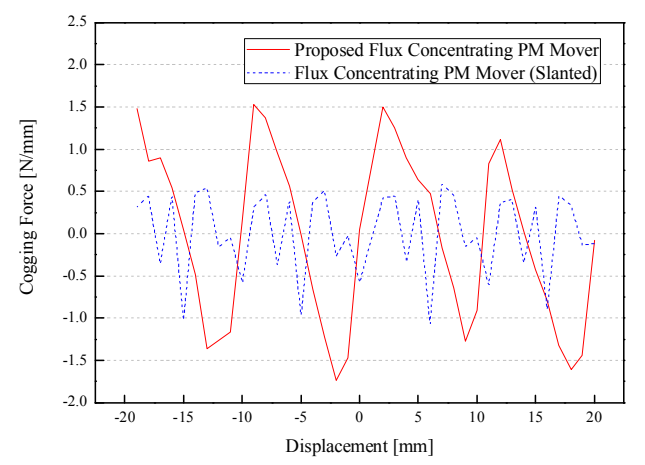

(d) Cogging force

Fig. 11. Comparison of flux concentrating PMs mover (Slanted) and proposed flux concentrating PMs mover 


$$
\begin{gathered}
W_{\text {max }}=\left(\emptyset_{a \delta}+\emptyset_{m \delta}\right)^{2} \cdot R_{\delta}+\left(\emptyset_{a \sigma}-\emptyset_{m \sigma}\right)^{2} \cdot R_{\sigma} \\
\quad+0.5\left(\emptyset_{a \delta}-\emptyset_{a \sigma}+\emptyset_{m \delta}+\emptyset_{m \sigma}\right)^{2} \cdot R_{m} \\
W_{\text {min }}=\left(\emptyset_{a \delta}-\emptyset_{m \delta}\right)^{2} \cdot R_{\delta}+\left(\emptyset_{a \sigma}+\emptyset_{m \sigma}\right)^{2} \cdot R_{\sigma} \\
+0.5\left(\emptyset_{a \delta}-\emptyset_{a \sigma}-\emptyset_{m \delta}-\emptyset_{m \sigma}\right)^{2} \cdot R_{m}
\end{gathered}
$$

The average force and force density can be calculated by difference of maximum and minimum magnetic energy.

$$
\begin{aligned}
& \Delta W=W_{\text {max }}-W_{\text {min }} \\
& F_{\text {ave }}=\Delta W / \tau_{p} \\
& F_{\text {den }}=F_{\text {ave }} / 2 \cdot \tau_{p} \cdot l_{z}
\end{aligned}
$$

\subsection{Comparison of flux concentrating slanted PMs and proposed model}

Fig. 11 presents the comparison of the flux characteristics, the flux density in air-gap under no-load, the cogging force under no-load and the relationship of force and weight of mover with magnets by different two types; flux concentrating slanted PMs mover and proposed flux concentrating PMs mover type. The model of flux concentrating slanted PMs mover shows the best results among the proposed models in the previous paragraph ; 5 . Selection of Surface Mounted and Flux Concentrating PMs Mover Types. Nevertheless, this model has a relatively lower flux performance than proposed flux concentrating PMs mover model by reason that continuity of flux is not satisfied sufficiently; the flow of the magnetic flux is interfered with a pair of magnets magnetized each other in the opposite direction. For such a reason, the slanted PMs mover can lead to a reduction the generator's force output occasionally because the windings and magnetic fields are no longer optimally aligned.

However, the flux concentrating slanted PMs mover model has superiority over the proposed flux concentrating PMs mover model in terms of only the force aspect by increasing the number of the magnets. Coulomb's Law supports the result that the force is inversely proportional to the square of the distance between different two poles. As a result, the flux concentrating slanted PMs mover model has a bigger force value due to the structure of two poles attached each other. The cross-section area of the mover with magnets in the compared two models is same completely except for their weight. An increasing in the number of magnet affects the weight of the mover, which in turn is related to the speed reduction of the mover. The ratio of the force to the weight is 3.2 to 1.2 . The proposed flux concentrating PMs mover model is studied in terms of flux density distribution in air-gap and cogging force under no-load through FE analysis. Compared to the interaction cogging force shown in Fig. 11(d), the cogging force in proposed flux concentrating PMs mover model is quite higher than that of the flux concentrating slanted PMs mover model. If the mover is slanted, the air-gap flux variation will also be reduced correspondingly. Eventually, the slanted mover to reduce the cogging force is successfully. Trade-off may be made between the flux linkage variation and the cogging force. So the slanted PMs mover does not adversely affect the flux variation very much. It is only influence on the cogging force by buried structure of magnets as geometrical variables. This result gives useful information in the design and evaluation of hybrid stepping machine through effective machine structure.

\section{Conclusion}

On developing a hybrid stepping linear oscillating generator, the buried forms of magnets are compared; one is surface mounted PMs mover and the other is flux concentrating PMs mover type. They are analyzed in symmetrical and asymmetrical stator cores in the former case, and in non-slanted and slanted PMs mover in the latter case. Different rectangular shapes of the magnets are investigated with respect to the magnetic flux in the air-gap, flux linkage and force characteristics. Due to concentration of magnetic flux, the flux concentrating PMs mover model shows better results than the surface mounted PMs mover model in terms of the flux linkage and force aspects. However, this model generates cogging force and it has a bad influence on the system. To overcome such difficulty, an improved force characteristic to minimize cogging force by slanting of magnets was found.

This study has presented a new structure reducing the number of magnets to half than the flux concentrating slanted PMs mover structure for hybrid stepping linear oscillating generator. This was investigated by analytical calculation considering leakage reluctances and was compared with FEA. At last, the proposed hybrid stepping generator with the flux concentrating PMs mover has a good performance in flux linkage, flux density in air-gap and weight of mover characteristics than the surface mounted PMs mover and the flux concentrating slanted PMs mover structure. Furthermore, it was necessary to examine how the electrical characteristics in accordance with slant-angle of the mover have an effect in the new flux concentrating PMs mover model.

\section{Reference}

[1] A. Cosic, J. Lindback, W. M. Arshald, M. Leksell, P. Thelin, and E. Nordlund, Application of a free-piston generator in a series hybrid vehicle, presented at the Proc. 4th Int. Symp. Linear Drive Ind. Appl., LDIA 2003, Birmingham, U.K., 8-10, 2003.

[2] Sungin Jeong, Modeling of linear oscillating generator for internal combustion engine, Jahresbericht 2009, IMAB. 
[3] J. Vining, T.A. Lipo, G. Venkataramanan, Design and Optimization of a Novel Hybrid Transverse / Longitudinal Flux, Wound-Field Linear Machine for Ocean Wave Energy Conversion, 2009 IEEE Energy Conversion Congress and Exposition.

[4] J. A. Güemes, A. M. Iraolagoitia, M. P. Donsión and J. I. Del Hoyo, Analysis of Torque in Permanent Magnet Synchronous Motors with Fractional Slot Windings, Proceedings of the 2008 International Conference on Electrical Machines.

[5] Jawad Faiz, Mahdi Ebrahimi-salary and Gh. Shahghplian, Cogging Force Alleviation in Linear Permanent Magnet Generators, IEEE AFRICON 2009, 978-1-4244-3919-5/09/\$25.00 C2009 IEEE.

[6] Sung In Jeong, Comparative Study of Linear Oscillating Generators, Dissertation an der Technischen Universität Braunschweig, 2015.

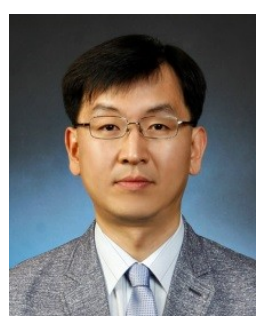

Sung-In Jeong He received B.S. and M.S. degrees in Electrical Engineering from Dongguk and Hanyang University, South Korea, respectively. After he received Dr.Ing. degree from Technical University Braunschweig, Germany. He is in the Daelim Motor, South Korea. His research and development field and interest included design, analysis and drive of electric machine for electric motorcycles. 